\title{
A Brief Discussion on Chinese Medicine Behaviour and Prescription Compatibility
}

\author{
Loh Cheng Toa Steven', Goh Xin Yi ${ }^{1 *}$ \\ 'NTU Chinese Medicine Clinic, Nanyang Technological University, Singapore
}

\section{Article Info}

\section{Article Notes}

Received:November 03, 2020

Accepted:December 11, 2020

\section{${ }^{*}$ Correspondence:}

Goh Xin Yi, NTU Chinese Medicine Clinic, Nanyang

Technological University, Singapore, Singapore;

Email: gohxy@ntu.edu.sg.

${ }^{\circ} 2020$ Yi GX. This article is distributed under the terms of the Creative Commons Attribution 4.0 International License.

\section{Abstract}

Chinese medicine (CM) is gaining popularity in recent years, prompting researchers' and clinicians' interest and effort into the makings and effects of it, especially after the announcement of World Health Organisation's incorporation of $\mathrm{CM}$ into the mainstream medical compendium, followed by the promising outcome brought about by the implementation of $\mathrm{CM}$ in the recent pandemic. Individual herb has complex properties, coming from its pharmacological properties and the Chinese medical principles of organdirected, taste and dynamic orientational behaviours. The use of individual herb in $\mathrm{CM}$ prescription is rare. Formula prescriptions predominantly consist of two ingredients or more. To fully reveal the effects of $\mathrm{CM}$ is a great challenge. The compound reaction of various herbs, the absorption and utility rate by the body, uniqueness of individual physique, sub-types of pathological behaviours and time-line progression at the healing process add on to the complexity of understanding the full effect of CM. Various theories such as pathophysiology guidance, pharmacokinetic-pharmacodynamic compatibility method, and Global Systems Biology for Integrative Genomics, Proteomics and Metabolomics, which interactively provide a more comprehensive and greater in-depth understanding, together with the consideration of development timeline, may shed more light to revealing the full picture of the effects of compatibility prescription.

\section{Background of Compatibility of Herbs in a Formular}

With thousands of years of application and experiences, traditional Chinese medicine (TCM) has been widely used in the modern society on disease prevention, treatment and health care. There are increasing interest and investments on the scientific research of Chinese medicine (CM) among medical and healthcare communities worldwide.

From the recognition of Nobel Laureate Tu YouYou's discovery on artemisinin treating malaria in $2015^{1}$, to the most recent World Health Organization's recognition of traditional medicine in its influential global medical compendium ${ }^{2}$, interest in traditional Chinese medicine (TCM) has reached a new height. It is to be noted, however, that artemisinin is an extract from the plant Artemisia apiacea, which can at most be considered an herbal medicine (or herbal extract) but falls short of being classified as CM. By definition, a traditional CM (TCM) prescription is (usually) a combination of various herbs with different properties that are grouped together to provide treatment for a certain condition based on the Basic Foundation Theory of $\mathrm{TCM}^{3}$. Compatibility herbs is a special feature of syndrome differentiation, which is a unique methodology of TCM diagnosis. The characteristic of compatibility herbs is the ability to adapt to the environment and changes ${ }^{4}$. Herbs compatibility can 
reduce the side effects, enhance efficacy of drugs, and is more suitable for the treatment of complex diseases.

Initiated by China at the 50's last century, much effort has been put into studying the biological properties of various herbs that are commonly used. Chinese herbal medicine has not been able to reveal the full effect of its properties in the clinical application and that there may be more to find out at prescription level in vivo ${ }^{5,6}$. Further justification of the clinical efficacy of TCM prescriptions may have to come from understanding the compatibility of herbs in a prescription and the mechanism of drug absorption in human body.

Therefore, the study of the compatibility of herbs within a prescription should not only focus on the drug quality and quantity changes (apparent compatibility), but instead pay more attention to the absorption of drug components, effects of the direct action of the substances in the body, and the intrinsic compatibility. Based on the overall metabolic changes due to the intrinsic compatibility of prescriptions and the study of ingredients (instituentsin vitro-and-in vivo), this could reflect the compatibility and efficacy of traditional CM.

$\mathrm{Li}^{7}$ reported that the TCM prescription interactive research includes: (1) the ranking of the compositions in four groups-Monarch, Minister, Assistant, Guide (also known as "Jun, Chen, Zuo, Shi"); (2) the compatibility from the seven emotions; (3) the four properties and five flavours of the herbs; (4) the dosage and ratio of interactive ingredients, which are important factors that will affect the efficacy and nature of the prescription; and (5) the environment, which will affect the efficacy of the prescription $^{8}$.

Based on pharmacodynamics, the study of the compatibility of the TCM prescription shows that amalgamation results in the formation of new substances change in chemical compositions and active ingredients. For example, common inorganic compounds, small molecules (such as volatile oils, alkaloids, flavonoids, saponins, etc.) and bio-macromolecules (peptides, proteins, glycopeptides, polysaccharides, etc.) are the basic ingredients of pharmacodynamic substances. Modern research can be carried out from different angles: one is the change of the composition of the drug in vitro after herbs compatibility; the other is that after the drug reaches the body, it undergoes biotransformation such as absorption, distribution, metabolism, excretion (ADME), and changes in the basis of pharmacodynamic substances $^{9,10}$. The expression of genes in the molecular level can also be used in the study of the compatibility of herbs in the prescription. For example, study on the pattern characteristics of gene expression profiles of cells, cardiomyocytes, and endothelial cells after intervention could be done, and it should focus on the effects of drugs on gene expression and regulation ${ }^{11}$.

\section{Efficacy and Toxicity}

The fundamental study of Chinese herbs is based on their individual properties such as: Herb Temperature (hot, warm, neutral, cool, cold etc.); the Four Qi (ascending, descending, floating, sinking); the Five Tastes (sour, bitter, sweet, acrid, and salty with extra tastes/properties of bland and astringent) and the Channel Tropism [Fei (Lungs), large intestine, stomach, Pi (Spleen), pericardium, Sanjiao, etc.). Herb-to-herb interactions constitute the basic principle of Compatibility Formulae, which include synergistic interactions such as Mutual Accentuation (Xiang Xu) and Mutual Enhancement (Xiang Shi); as well as antagonistic interactions such as Mutual Counteraction (Xiang Wei), Suppression (Xiang Sha), Antagonism (Xiang $\mathrm{Wu}$ ), Incompatibility (Xiang Fan) ${ }^{12}$.

TCM classic "Shen Nong's Herbal Classic" (Shen Nong Ben Cao Jing0) ${ }^{12,13}$ summarizes the Chinese herbal medicine formulae into 7 compatible relationships: "Single effect, Mutual Reinforcement, Mutual Assistance, Mutual Restraint, Mutual Detoxification, Mutual Inhibition, Antagonism", indicating the change in drug efficacy and toxicity after the compatibility of TCM. The changes from the compatibility of herbal medicine can be summarized as four aspects: enhancing efficacy, reducing toxicity, reducing efficacy, and enhancing toxicity. Wang ${ }^{14}$ used Nuclear Magnetic Resonance (NMR) in the study of the metabolites of the Paeonia lactiflora (Baishao )and Toosendan Fuctus (Chuanlianzi). The group with both Paeonia lactiflora and Toosendan Fuctus as compared to the group with only Toosendan Fuctus has a relatively milder change in urinal metabolites (formic acid, glucose, and lemon acid, succinic acid, 2-ketoglutaric acid (2-OG), creatine/creatinine, lactic acid, glutamine, trimethylamine $\mathrm{N}$-oxide (TMAO)). This shows that Paeonia lactiflor has a certain neutralizing effect against the toxicity of Toosendan Fuctus.

$\mathrm{Ni}$, et $\mathrm{al}^{15}$ in the study of the pharmacokinetics of Zuojin Pill in rats, intragastrically administered the decoction of Zuojin Pill, berberine (Huanglian) and tetradium ruticarpum (Wuzhuyu) into SD rats. High performance liquid chromatography (HPLC) method was used to determine and calculate berberine and rutaecarpine. It was found that after the administration of Zuojin Pill, berberine and ruticarpum reached a higher concentration at about $0.5 \mathrm{~h}$, in which ruticarpum can sustain at higher concentration to have anti-inflammatory, antibacterial functions and ruticarpum can better inhibit gastrointestinal motility to protect gastric mucosa.

Zhong $^{16}$ and other research reports on the effects of Chinese materia medica (CMM) compound in recent years, with the compatibility of two TCMs, compatibility 
of traditional Chinese prescriptions, and compatibility of monomer components, concluded that the compatibility of CM mainly achieve its effect of reducing toxicity and increasing efficiency.

\section{Research Approach}

Deng $^{5}$ and Zhang ${ }^{11}$ both in their reports highlighted the importance of comparing both the direct combination form based on prescription theories versus the combination form based on efficacy. The mechanism of the latter may on one hand lead to further strengthening of conventional combination theories, and on the other hand lead to development of new combinations that are relatively effect-specific and research viable. However, in the midst of seeking scientific evidence to prove efficacies of Chinese medicine, it is imperative that Chinese Medicine theory should not be lost in the process, otherwise any results derived from these studies may be detached from reality and found themselves useless in the field of actual clinical application $^{17}$.

At present, metabolomics plays an important role in the discovery of biomarkers ${ }^{18,19}$, early diagnosis of diseases ${ }^{20}$, pathogenesis ${ }^{21,22}$, and evaluation of pharmacological effects $^{23}$. All these have been used in the study on malignant tumors $^{24,25}$, heart disease ${ }^{26}$, diabetes ${ }^{27}$ and other major diseases research. Using metabolomics technology, Liu, et $\mathrm{al}^{28}$ discovered that Huanglian Jiedu Decoction has a good intervention effect on two heaty syndromes. Zhao, et $\mathrm{al}^{29}$ studied the effects of Erzhi Pill on endogenous metabolites in natural aging mice through urinary metabolomics analysis, and found that there are 36 changes in mouse urine metabolites, which can improve immunity from the body. It regulates nerve function, regulates endocrine, promotes metabolism, anti-oxidation, and removes free radicals. It also delays aging through multiple levels, multiple pathways and multiple targets.

$\mathrm{Qu}$, et $\mathrm{al}^{30}$ uses the formulae "Banxia Xiexin Decoction" as an example and artificially synthesized 4 components (saponins baicalin, berberine, ginsenoside and glycyrrhizic acid) from the decoction to make immunized animals, clones and other immunized models to study the interaction and biological pathway of the formulae components in the body. Monoclonal hybridoma cell lines were established by cell fusion, monoclonal antibodies were prepared, and corresponding immunoassay methods were established to analyze the in vivo processes and compatibility of the main components of "Banxia Xiexin Decoction". It is also proposed that the immunoassay technique (IAT) has high specificity compared with traditional gas chromatography (GC), HPLC or liquid chromatography-mass spectrometry (HPLC-MS). IAT has high sensitivity, is convenient and fast, and includes various technical modes such as radioimmunoassay (RIA), enzyme immunoassay (EIA), fluorescent immunoassay
(FIA), chemiluminescence immunoassay (CIA) and solid phase immunosensor, etc.

\section{Current Research Methods}

Wang, et $\mathrm{al}^{31}$ used principles from the pharmaco metabolomics of chinmediformulae, and PK-PD binding theory on the direct effect of prescription compatibility on the overall effect in the body, to analyse the compatibility of the composition of "Yinchenhao Decoction". Using UPLC/ MS technology, 45 compounds were identified from in vitro samples of "Yinchenhao Decoction" concoctions in vivo to reveal the pharmacological properties and compatibility of ADME/T.

$\mathrm{Xu}^{32}$ suggested that many experimental studies had neglected the theoretical connotation of TCM in combination with prescriptions. In order to study the compatibility mechanism of prescriptions, a research method of "targeted drug combination of disease pathophysiology guidance" was proposed. This theory uses the specific diseasesyndrome- target prescription combination approach to understand the multi-targeted effect of Chinese herbal compound in the body. This theory suggests that the "functional unit" should be highlighted when studying the drug target, such as (1) cell function unit: pathway signal/ metabolic pathway; (2) tissue and organ function unit: vascular smooth muscle-nerve-humoral regulation; (3) whole body function unit of depression: Central nervous system and endocrine system, the combination of the thalamus-pituitary-adrenal (HPA) axis.

Wang, et $\mathrm{al}^{33}$ reports the focus of research on the prescription theory in the prescription itselfand its dynamic process (pharmacokinetics). It is proposed that the research method can together focus on the pharmacokineticpharmacodynamic compatibility of the "chemical composition of CM" and "intestinal micro-ecological balance" in the syndrome-prescription TCM theory. Five steps were proposed to use the pharmacodynamics(PD) and pharmacokinetics(PK) biomarkers parameters for analysis, that is, to explain the compatibility of CM herbs from the intrinsic requirements of the whole TCM theory.

Prescription Compatibility in Molecular, Genomic and Systemic Studies for Various Illnesses

\section{AIDS}

In July 2006, the US Food and Drug Administration (FDA) approved the launch of Atripla, a new anti-AIDS drug, which is a combination of three FDA-approved drugs, Viread, Emtriva and Sustiva, for a "cocktail therapy" combination. This fully shows that the mainstream international society also recognizes the limitations of the Western single drug model, and begins to learn from the CM mode of compound drug use ${ }^{34}$. American professor Leory Hood first integrated genomics, proteomics and computation into what is known 
as the Integrative Systems Biology ${ }^{35}$, the significance of which is to study the inter-relationship between various elements in the biological systems ${ }^{36}$. Based on the established metabolomics approach, Professor Nicholson proposed Global Systems Biology for Integrative Genomics, Proteomics and Metabolomics ${ }^{37}$. Attachment: Figures 1-3 ${ }^{38}$.
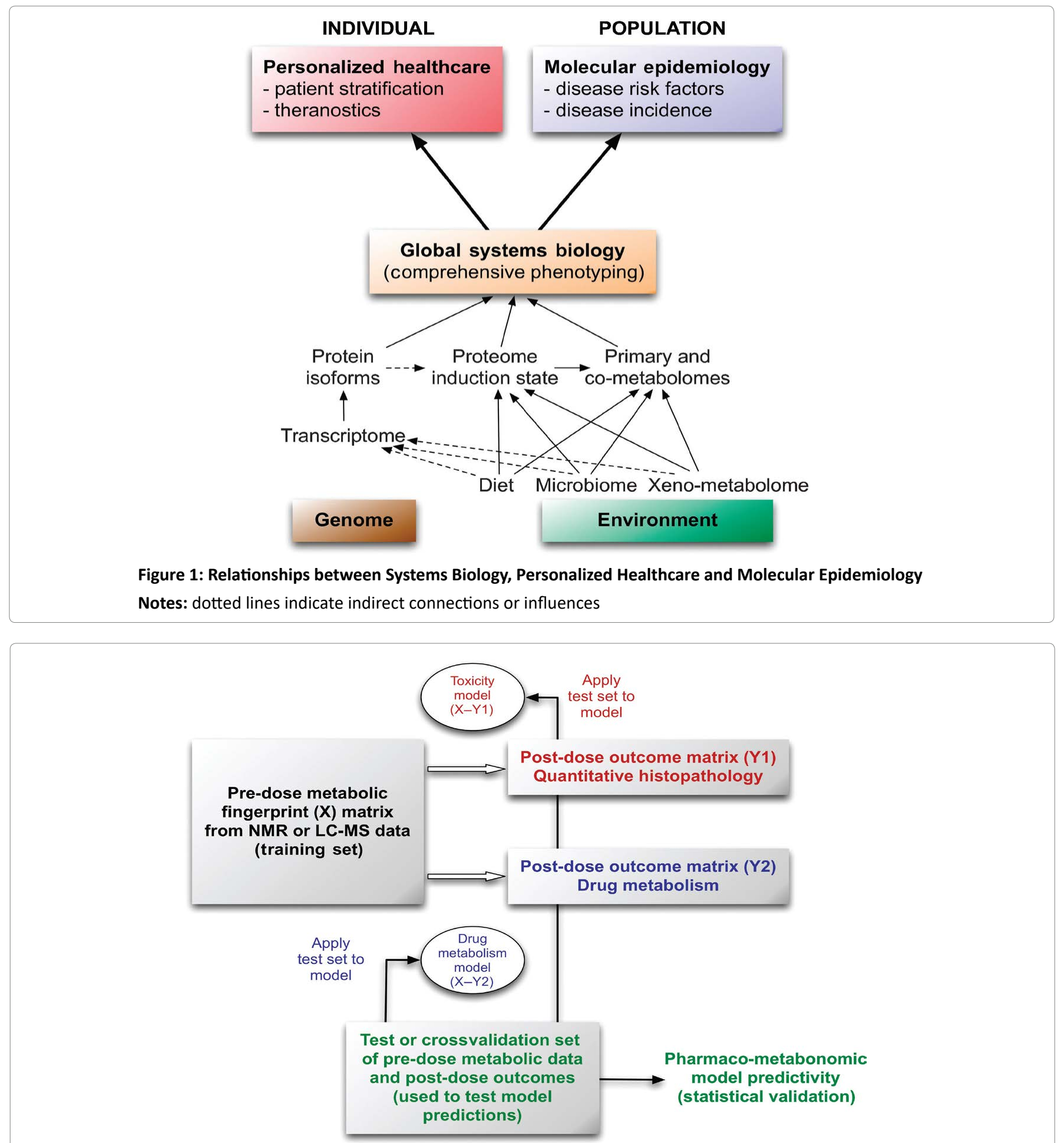

Figure 2: Pharmaco-Metabonomic Modelling Procedure

Notes: spectroscopic data on pre-dose metabolic fingerprints (X matrix) from biofluids such as urine and plasma are statistically linked to outcome [quantitative toxicity (Y1) drug metabolism (Y2) matrixes] of a drug intervention via multivariate statistics such as partial least squares methods. Typically, $20 \%-50 \%$ of all data is used in the training set construction. The predictive power of the models is then tested using a test set or a cross-validation set to assess model robustness. It is also possible as an additional test to avoid overfitting of data, to deliberately permute the training set matrixes to induce a false model that should have a very low predictive capability. 


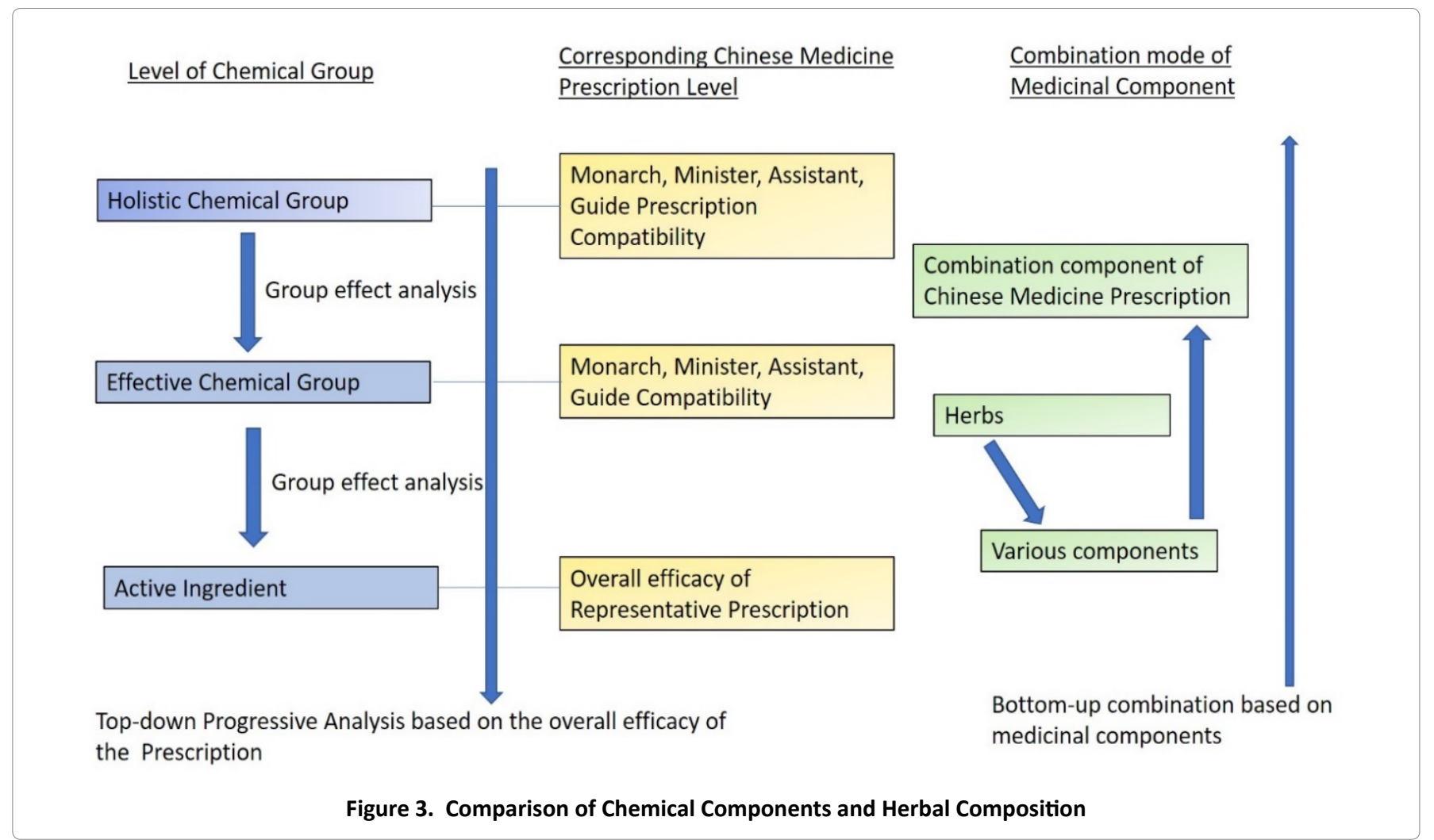

$\mathrm{Li}$, et $\mathrm{l}^{39}$ pointed out that the usage of a combinatory of the effective components is different from the "cocktail therapy" adopted by (highly active antiretroviral therapy ?HAART) in the research and methodology for treating AIDS. The latter is made up of several antiviral chemical monomers, whose main target is the human immunodeficiency virus (HIV), and the "combinatory of effective components group" belongs to the CM treatment system, which complies with the compatibility rules of "Jun, Chen, Zuo, Shi" and "Seven Emotions" for targeted treatment. The molecular basis and targeted treatment from this system could be multiple. Using the "Kangai Baosheng Pills" for treatment of AIDS as an example, this concoction consists of Astragalus ?Huang qi) that can regulate immunity, and Viola philippica (Zihua Diding) which inhibits the virus. The compounded effect produced by the complimentary use of these 2 herbs in the concoction is obviously higher than that of the single-flavoured medicine or from the disassembled prescription.

\section{Cancer}

Hepatocellular carcinoma (HCC) is the most common primary liver malignancy and is a leading cause of cancerrelated death worldwide. Progressive development of anti-cancer drugs such as kinase inhibitors Sorafenib and Lenvatinib, or monoclonal antibody Ramucirumab, tend to exhibit low response and high toxicity.

In the competing endogenous RNA (ceRNA) regulation mechanism, MicroRNA (miRNA) response element (MRE) is the binding site of mRNA and miRNA,

serving as a natural molecular sponge for miRNA, miRNA in turn inhibits its target by binding with MRE, causing translational inhibition or mRNA degradation. During such regulation process, each miRNA can regulate multiple RNA targets. Large numbers of miRNA binding sites exist in different types of RNA transcripts, indicating that multiple RNA transcripts containing miRNA binding sites can be mutually regulated via shared competition. This further illustrate that the synergistic effect of miRNA and RNA transcripts is the main source of ceRNA regulation ${ }^{40}$.

LI Lu ${ }^{41}$ explore the anti-tumor mechanism of traditional Chinese medicine based on ceRNA regulation. In the occurrence and development of HCC, the mechanism of competitive endogenous RNA (ceRNA) plays an important role, in which miRNA is the core of its regulatory functions. Li reported that under the action of miRNA response element (MRE), mRNA, pseudogene transcripts, Long non-coding RNAs (lncRNA), Circular RNA (circRNA) and others competitively combine with miRNA to form a ceRNA regulatory network. Although researches on this mechanism at present it is still at the exploratory stage, it may well be an important anti-tumor mechanism for the diagnosis, treatment and research of tumor in traditional Chinese medicine.

\section{Uterine Fibroids}

Zheng $^{42}$ explored the molecular mechanism of 
concoction formula Modified Guizhi Fuling Wan in 72 specific pathogen free (SPF grade) SD rats with uterine fibroids. The experiment uses HE staining to observe the morphological changes at the uterus, micRNA gene chip to detect the expression profile of uterine micRNA gene; bioinformatics methods screening of micRNA for Differential expressions. Gene function enrichment was then used to predict the possible signaling pathways in rats with uterine fibroids under the influence of Guizhi Fuling Wan.

Gene function enrichment analysis indicated that four signaling pathways were closely related to uterine fibroids. They were MAPK signaling pathway, Wnt

signaling pathway, mTOR signaling pathway and VEGF signaling pathway. The result indicated Chinese medicine Modified Guizhi Fuling Wan affected the expression profile of micRNA in rat model of uterine fibroids induced by estrogen and progesterone, suggesting its involvement in a variety of biological processes such as signal transduction and gene regulation in the treatment of uterine fibroids.

\section{Coronary heart disease}

Coronary heart disease is a disease with complex trait, multi-gene heredity and environmental factors. Blood stasis syndrome is one of the differential syndrome in the context of TCM. Epigenetics being a hotspot in the field of cardiovascular research, its mechanisms can explain the interaction between genes and the environment. The occurrence and development of coronary heart disease may be closely related to the abnormal methylation of DNA, while modifications are reversible.

$\mathrm{Li}$ et $\mathrm{al}^{43}$ reported from research findings that Increased or high expression of MMP-9 will accelerate the development of atherosclerosis.

Ghaznavi et al. ${ }^{44}$ showed that the CHD patient group ATPbinding cassette transporter A1 (ABCA1) gene methylation frequency was significantly higher than control group, suggesting ABCA1 Gene methylation is an important risk factor for the development of coronary artery disease.

Zhou et al. ${ }^{45}$ have shown that formula Chinese medicine Zhizi Chuanxiong Capsules can treat atherosclerosis by regulating both abnormal hypermethylation genes and hypomethylation genes. The altered methylation genes are involved in the activation process of protein kinase $C$, inflammation pathway, MAPK signal transduction pathway and vascular endothelial growth factor signal transduction pathway.

\section{Discussion}

One intrinsic difference between Western and CM is the approach towards perception and treatment of sicknesses. Western drug is highly standardized and able to pin-point pathogens and zoom in to precise causative location to as far as genomic level. Whereas CM adopts a more broad-based and holistic approach, which in the midst of treating the ailment, also includes maintaining the patient's quality of life and improving overall wellbeing. One of the very reasons in prescription compatibility is to reduce side-effects while enhancing treatment efficacy. This nourishment and preventive maintenance effect is similar to enhancing immunity in the western science perspective.

This report has not covered prescription compatibility in the genomic aspect as studies in this area are still quite limited. While it is imminent to investigate the genomic impact, it may be premature to do so at this juncture for prescription compatibility on proteomic studies are not completely covered and genetic science itself is yet to be fully chartered.

In many a case, studies investigated one or few aspects of the full spectrum, and many of the outcome measures that may be considered important were absent. There was a shortage of clinically relevant event outcomes and especially with little or no measurement of patients' quality of life.

\section{Conclusion}

Due to the nature of Chinese herbal medicine, and its use in combination with various components (ranking of the compositions), knowing its main efficacy for a particular condition is attainable, but to fully understand all relationships and cause-and-effects it has on the body remains a great challenge. Bearing in mind that each herb is multi-targeted, multilevel and multi-link in effect; various herbs in amalgamation will produce a more complex second tier effect; in-vivo absorption will produce a third-tier effect. Notwithstanding the fact that individual difference in age and physique plays an equally important part in the reaction and efficacy of the medicine. Another characteristic in the application of $\mathrm{CM}$ is the dynamic adjustment of prescription during the process of treatment, allowing it to capture the finest change in the body system and target those changes accordingly. In order to avoid missing the forest for the trees, a bird's eye view and evaluation for the entire timeline of treatment would be crucial.

Studies and methodologies proposed by various experts such as: (1) "targeted drug combination of disease pathophysiology guidance"; (2) the pharmacokineticpharmacodynamic compatibility method; (3) the Global Systems Biology for Integrative Genomics, Proteomics and Metabolomics, synergistically integrated to provide a wider scope, fuller details, and development time-line, may perhaps come close to revealing the full picture of the effects of compatibility prescription. 
Establishing a framework that is unanimously accepted by the research community on the study of prescription compatibility in CM (or any medicine for the matter), may help to bridge differences between Chinese and western medicine, synergize philosophies of east and west on drug applications and shed more light to a current predominantly random and scattered research environment.

\section{Author Contributions}

Loh Cheng Toa Steven: writing-original draft, review. Goh Xin Yi: writing-review, editing. All authors have read and approved the final manuscript.

\section{References}

1. Tu Y. The discovery of artemisinin (qinghaosu) and gifts from Chinese medicine. Nat Med. 2011; 17: 1217.

2. Cyranoski D. The big push for Chinese medicine. Nature. 2018; 448450.

3. Deng ZJ. TCM Formulary. 1st ed. Beijing: China Press of Traditional Chinese Medicine. 2003; 10-13.

4. Chen JP, Wu WK, Zhang MS, et al. Study on the ideology and methodology of the compatibility of traditional Chinese medicine compound. Chin J Exp Tradit Med Formul (Chin). 2000; (01): 1-4.

5. Deng ZY, Wang MJ, Zhang JQ, et al. An Cough formulae compatibility qualitative and quantitative research. Shandong J Tradit Chin Med (Chin). 2018; 37: 456-459, 493.

6. Mao ZP, Liu HN, Xiong YX, et al. Data analysis study of usage of formulae compatibility. J Jiangxi Univ Tradit Chin Med (Chin). 2017; 29: $118-121$.

7. Li Z, Rong YY, Wang SM, Wu CW. Formulae compatibility study. Chin J Exp Tradit Med Formul (Chin). 2015; 21: 223-226.

8. Gao XM. Chinese Materia Medica. 1st ed. Beijing: China Press of Traditional Chinese Medicine. 2002; 21-30: 42-43.

9. Fan FJ, Hong WX, Song JL, et al. Discovery and research on the formulae visualization compatibility. Chin J Biomed Engin (Chin). 2016; 35: 764-768.

10. Fan DM, Huang YQ. Current research on formulae compatibility. Strait Pharm J (Chin). 2018; 30: 20-23.

11. Zhang Y, Xie YM. Genetics analysis of Chinese herbal medicine. Chin Arch Tradit Chin Med. 2001; (05): 426-427.

12. Deng ZJ. TCM Formulary. 1st ed. Beijing: China Press of Traditional Chinese Medicine. 2003; 17-19

13. Gao XM. Chinese Materia Medica. 1st ed. Beijing: China Press of Traditional Chinese Medicine. 2002; 36-41.

14. Wang XJ. Metabonomics study on Chuan Lian Zi toxicity and compatibility [D] Hefei:Anhui Medical University,2011.

15. Ni JX, Lin YH, Chen MZ. Pharmacokinetics analysis of the compatibility of Zuojin Pill. China Mod Med (Chin). 2012; 19(05): 19-20

16. Zhong XY, Huo WZ, Gong $Q$, et al. Research status of pharmacokinetics of traditional Chinese medicine compatibility. Chin Herb Med (Chin) 2013; 44: 3084-3088.

17. Wu JF. Formulae compatibility modern scientific research. J Liaoning Coll Tradit Chin Med (Chin). 2014; 16: 217-219.

18. Sato Y, Suzuki I, Nakamura T, et al. Identification of a new plasma biomarker of Alzheimer's disease using metabolomics technology. J Lipid Rese. 2012; 53: 567-576.
19. Huang Z, Lin L, Gao Y, et al. Bladder cancer determination via two urinary metabolites: a biomarker pattern approach. Mol Cell Proteom. 2011; 10(10): M111.007922-M111.

20. Roberts MJ, Schirra HJ, Lavin MF, et al. Metabolomics: a novel approach to early and noninvasive prostate cancer detection. Kore Urol. 2011; 52: 79-89.

21. Patti GJ, Yanes O, Shriver LP, et al. Metabolomics implicates altered sphingolipids in chronic pain of neuropathic origin. Nat Chem Biol. 2012; 8: 232

22. Brockmöller SF, Bucher E, Müller BM, et al. Integration of metabolomics and expression of glycerol-3-phosphate acyltransferase (GPAM) in breast cancer-link to patient survival, hormone receptor status, and metabolic profiling. J Proteome Res. 2012; 11: 850-860.

23. Kaddurah-Daouk R, Kristal BS, Weinshilboum RM. Metabolomics: a global biochemical approach to drug response and disease. Ann Rev Pharmacol Toxicol. 2008; 48: 653-683.

24. Zhou L, Wang $\mathrm{Q}$ Yin $\mathrm{P}$, et al. Serum metabolomics reveals the deregulation of fatty acids metabolism in hepatocellular carcinoma and chronic liver diseases. Anal Bioanal Chem. 2012; 403: 203-213.

25. Patterson AD, Maurhofer O, Beyoglu D, et al. Aberrant lipid metabolism in hepatocellular carcinoma revealed by plasma metabolomics and lipid profiling. Cancer Res. 2011; 71(21): 6590-600.

26. Rhee EP, Gerszten RE. Metabolomics and cardiovascular biomarker discovery. Clin Chem. 2012; 58: 139-147.

27. Leslie RD, Beyan H. Metabolomics makes a mark: early changes associated with autoimmune diabetes. Diabetes. 2011; 60: 26882690.

28. Liu CF, Wang N, Liu SM, et al. Metabolomics sudy of Huanglian Jiedu Tang on fever syndrome. J Shandong Univ Tradit Chin Med (Chin). 2011; 35: 60-63.

29. Zhao Y, Su W, Zhang QY, et al. Effects of Erzhi Pill on Urine metabolites in naturally aging mice. Pharmacol Clin Chin Mater Med (Chin). 2011; 27: 3-5.

30. Qu HH, Zhao Y, Wang QG. Compound compatibility mechanism based on Chinese medicine moelecular monoclonal antibodies. Chin J Integr Tradit West Med (Chin). 2012; 32: 1416-1419.

31. Wang XJ, Zhang BL. Drug metabolomics and scientific compatibility of prescriptions compatibility. China J Chin Mater Med (Chin). 2010; 35: $1346-1348$

32. Xu YT. Modern scientific study of formulae compatibility. Chin Herb Med (Chin). 2015; 46: 465-469.

33. Wang ZG, Hu HL, Lan K, et al. Study on the Compatibility of traditional Chinese medicine compounds based on pharmacodynamicspharmacokinetics method based on metabolomics and prescription theory. Chin Herb Med (Chin). 2009; 40: 169-172.

34. Luo GA, Liang QL, Liu QF, et al. Integrating chemical systemic study of systemic biology--study on Chinese herbal compatibility and mechanism of Chinese medicine compounds. Modern Tradit Chin Med Mater Med-World Sci Technol (Chin). 2007; 10-15, 24.

35. Hood L, Tian Q. Systems approaches to biology and disease enable translational systems medicine. Genomics, proteomics \& bioinformatics. 2012; 10(4): 181-5.

36. Hood L. Systems biology: integrating technology, biology, and computation. Mechan Age Develop. 2003; 124: 9-16.

37. Nicholson JK, Wilson ID. Understanding 'global' systems biology: metabonomics and the continuum of metabolism. Nat Rev Drug Disc. 2003; 2: 668 .

38. Nicholson J. Global systems biology, personalized medicine and molecular epidemiology. Mol Syst Biol. 2006; 52 
39. Li YP, He LS, Zhao Y, et al. Study status and new thoughts on Chinese materia medica formula used for AIDS treatment. Chin Herb Med (Chin). 2014; 45: 303-307.

40. Swain AC?Mallick B. mi RNA-mediated "tug-of-war" model reveals

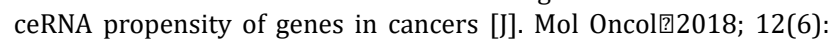
855-868.

41. Li L, Liu YH, Tang CC, et al. Research progress on competing endogenous RNAs regulation mechanism in the development of hepatocellular carcinoma and TCM interventions [J]. SH J TCM. 2020; 54(04): 58-64.

42. Zheng DX, Liu JY, Chen RF, et al. Molecular Mechanism of Modified Guizhi Fuling Wan Intervention on Uterine Fibroids in Rats Based on
micRNA Expression Profiling [J/OL]. Chinese Journal of Experimental Traditional Medical Formulae. 1-7[2020-0928]. https://doi. org/10/13422/j.cnki.syfjx.20202323.

43. Li M, Sun Y, Qiu RJ et al. Research Progress on the relation of DNA methylation and Coronary Heart diseases related TCM syndromes [J]. Chin J Integr Trad WMed. 2019; 39(4): 509-512.

44. Ghaznavi H, Mahmoodi K, Soltanpour MS. A preliminary study of the association between the ABCA1 gene promoter DNA methylation and coronary artery disease risk [J]. Mol Biol Res Commun. 2018; 7(2): 59-65.

45. Zhou QB, Wu LQ, Zhang Y, et al. Effects of Zhizi Chuanxiong Capsule on the abnormal methylation in rabbits with atherosclerosis[J]. Chin J IntegrMed. 2018; 24(7): 512-517. 